\title{
ON THE EQUATION OF STATE OF HYDROGEN AND ITS USE IN MODELS OF MAJOR PLANETS
}

\author{
R. S. HAWKE* \\ University of California Lawrence Livermore Lab.**, Calif., U.S.A.
}

\begin{abstract}
Since Jupiter and Saturn are considered to be composed primarily of hydrogen, its pressuredensity equation of state is needed for computational models of their interiors. Until recently, experimental data were limited to $20 \mathrm{kbar}$ statically and $40 \mathrm{kbar}$ dynamically. Since the majority of a major planet is at a pressure in excess of this, there were only theoretical calculations available for modeling.

Wigner-Seitz type calculations have been shown to be accurate at determining the equations of state of the alkali metals. Hence, it has been assumed that the equation of state of metallic hydrogen can be calculated in the same way with fair confidence. However, the molecular hydrogen equation of state has been much more ellusive. The many attempts at modeling the interatomic forces have led to rather scattered pressure density relationships.

The planetary model situation is further complicated by the expectation that the transition from the molecular to the metallic phase will be in conjunction with a relatively large density change.

Recently, data from new experiments have become available; in one case up to $8 \mathrm{Mbar}$. The data are not in disagreement with many calculations on hydrogen, but the resolution is not yet adequate to determine accurately and confidently, the pressure and the densities of the molecular to metallic phase transition. The accuracy of these parameters in turn affect the models of the planetary interiors, such as the radii of the metallic sphere and high density core.

This paper will discuss the details of these relations and the possible affects of the speculative properties of metallic hydrogen.
\end{abstract}

\section{Introduction}

First I would like to discuss the theoretical and experimental status of the Equation of State (EOS) of molecular and metallic hydrogen. Second, I would like to discuss their application to the models of Jupiter and Saturn. And finally, I would like to mention some of the proposals of the properties of metallic hydrogen as they apply to the major planets or at least Jupiter and Saturn.

For nearly 40 years it has been expected that molecular hydrogen will transform to the metallic phase at a pressure of the order of a Megabar (Mbar) (Wigner and $\mathrm{Hu}$ nington, 1935). Until recently, pressures of that magnitude were not attainable in the laboratory in a form that could allow measurements of the EOS and properties of soft and low density materials. This was especially true of hydrogen. Direct experiments on hydrogen at very high pressures, have only recently been feasable. The existence of metallic hydrogen in the interiors of the major planets is well established (Ramsey, 1950; Kronig et al., 1946; Abrikosov, 1954; Demarcus, 1958; Peebles, 1964; Smoluchowski, 1967; Hubbard, 1968). In fact, in the case of Jupiter, nearly all of the mass is in the metallic phase. As far as models for major planets go, the largest uncertainties about hydrogen have been the EOS of its molecular phase. Figure 1 shows the pressure in Mbars plotted as a function of density in $\mathrm{g} \mathrm{cm}^{-3}$, at $0 \mathrm{~K}$, for many theoretical cal-

* On leave at the Max-Planck Institut für Festkörperforschung, Stuttgart, West Germany.

** Work performed under the auspices of the U.S. Atomic Energy Commission. 
culations. The right most curves above about a megabar, are the calculations for the EOS of the metallic phase, while the left most curves at lower pressure are those of the molecular phase. The metallic calculations vary less than $10 \%$ in density. The molecular calculations vary by as much as $50 \%$. The transition from the molecular to the metallic phase is expected to be accompanied by a large change in density (20 to $50 \%)$. The range in pressure at which the transition is expected $\left(P_{t}\right)$, varies from a little less than a megabar to many megabars. The effects of these uncertainties will be considered as they influence the planetary models. There then remains two further interesting questions about hydrogen. First is the question of the solvability of helium in the various phases and throughout the pressure range of the planetary interiors, say 0 to

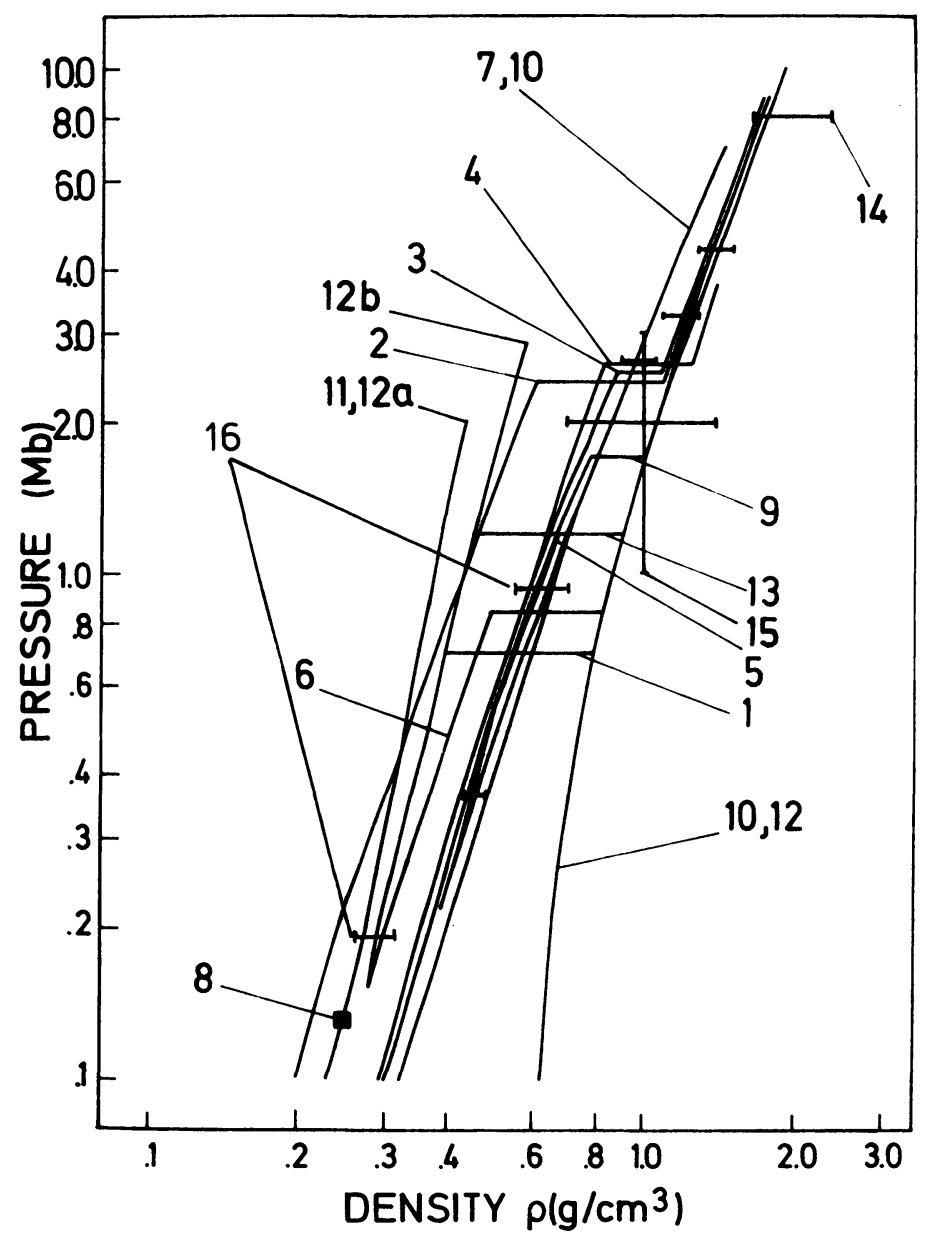

Fig. 1. The pressure as a function of density at $0 \mathrm{~K}$ for many theoretical calculations. Curves: 1 - Kronig et al. (1946), 2 - Abrikosov (1954), 3 - DeMarcus (1958), 4 - Schneider (1969), 5 - Ross (1970), 6 - Neece et al. (1971), 7 - Liberman (1972), 8 - Dick (1972), 9 - Hoover et al. (1972), 10 - Trubitsyn (1966), 11 - Etters et al. (1971), 12 - Pollack (1972), 13 - Ostgaard (1972), 14 - Grigoryev et al. (1972), 15 - Hawke et al. (1972), 16 - Van Thiel et al. (1972). 
40 Mbar. This subject just began to be investigated in the laboratory (Street, 1973) and has upset the simplifying assumptions that have aided modelling (Smoluchowski, 1973). This question is already being considered and will not be included here. The other remaining question concerns the possible effects of the unusual properties that metallic hydrogen might have. In particular, what could be the effects of the hoped for high temperature superconducting transition temperature (Ashcroft, 1968; Schneider, 1969) and the even more hoped for metastability of the metallic phase (Schneider, 1969; Brovman et al., 1971).

\section{The Equation of State of Hydrogen}

Metallic hydrogen is expected to be the simplest of all metals. To calculate its EOS, people have used increasing refinements of the Wigner-Sietz method (Wigner and Sietz, 1933). This method leads to the energy, per atom, as a function of atomic volume by assuming three contributions, (1) the ground state energy of an electron in a periodic field caused by the hydrogen nuclei and other electrons, (2) the Fermienergy, which represents the average energy of motion per electron above the ground state due to the energy band being half filled and, (3) a correction for electron exchange and correlation of their positions. The volume derivative then gives the pressure-volume EOS. Wigner and Hunington (1935) were the first to use this method and agreement has been fairly good since then as indicated in Figure 1. One of the refined calculations (Neece et al., 1971) applied to the alkali metals, as a check, was found to be quite successful. Schneider has made extensive calculations of the possible crystal structures and obtained a slightly denser EOS.

In contrast to the relative simplicity and agreement of the calculations for metallic hydrogen, the case for molecular hydrogen is less precise. This is partly because the iterations have not yet been completely described from first principles by reason of the extreme complexity; hence, more approximate calculations are used. Part of the complication is due to the large change in the intermolecular spacing that occurs as the pressure increases from zero to a few megabars. At room pressure the spacing between the molecules is large compared to the atomic spacing within the molecule, but these distances become comparable in the high pressure region. Generally the attractive and repulsive forces are based on two types of approaches, LennardJones and Buckingham. The Lennard-Jones form takes the repulsive potential to be proportional to the inverse twelfth power of the spacing, while the Buckingham (exponential-six) assumes the repulsive potential to be proportional to the inverse exponent. Both methods take the attractive potential as proportional to the inverse sixth power. The differences in the results are due to the choice of different coefficients in the relationships and sometimes added terms. Usually these calculations are made to agree with the experimental data of Stewart (1956), which give the pressure volume relationship up to 20 kbars. Pollack et al. (1972) used the Domb-Slater method with both types of repulsion and got results that are stiffer than others in both cases. Recently there have been shock wave experiments of two types, plane and cylindrical, 
and a magnetic technique used to compress hydrogen. In Figure 1 the shock results of Dick (1972) and van Thiel et al. (1972) and the multiple shock results of Grigoryev et al. (1972) and the magnetic work of Hawke et al. (1972) are shown. Due to the low initial density and great compressibility of hydrogen, single shock experiments are limited to pressures of a couple hundred kilobars. From preliminary analysis of the plane wave shock results, it has been found that good agreement can be made (van Thiel et al., 1972) with the exponential-six model calculations of Ross (1970). It is nice to note that the agreement between Ross (1970), Trubitsyn (1966), and DeMarcus (1958) is good, as many of the planetary models have used the later two. The maximum discrepancy is about $6 \%$ in density. The experimental data of Grigoryev et al. (1972) are also in agreement. There is some reservation about the support that data gave to the understanding of hydrogen's EOS (Al'tshuler et al., 1973). Also the pressure was apparently calculated and there is no support for the lack of uncertainty in the results. In any case, the data leave the pressure of the transition open over a range of at least a couple of megabars, and a $30 \%$ or more uncertainty in the density of the molecular phase at the transition pressure $\left(D_{t}\right) .{ }^{*}$ The magnetic compression has not yet been precised enough to reduce the uncertainties. Effort is being made to measure the pressure and volume and also to measure the metallization directly and electrically. The shock experiments also leave an open limit on the upper end of $P_{t}$ but indicate a lower limit of 1.4 Mbar. (In summary, of the several experiments done in the megabar range, none can pin down $P_{t}$ nor the molecular transition density.) As of yet, no one has reported static experiments beyond $20 \mathrm{kbar}$, although laboratories in the U.S. and Russia are striving to that end. It is extremely difficult and likely to be several years before a pressure in excess of a megabar will be statically exerted on liquid or solid hydrogen with the facility to make EOS or conductivity measurements.

\section{Effect of Uncertainty in EOS on Planetary Models}

The fortunate part of the situation is that the overall dependence of planetary models on the EOS of hydrogen is about the same order of magnitude as the uncertainty. Demarcus (1958) extrapolated two EOS's of molecular hydrogen that differed by $2 \%$ at $20 \mathrm{kbar}$. In the megabar range the simple extrapolation of Stewart's data is shown as curve 3 in Figure 1. The alternate stiffer form of molecular hydrogen is similar to curve 6 with the phase transition at $1.9 \mathrm{Mbar}$ instead of $2.5 \mathrm{Mbar}$ as for the softer form. The density difference at $1 \mathrm{Mbar}$ is nearly $10 \%$. The effect of the change in EOS on the planetary model was not stated as it was concluded that the alternate EOS was the most suitable at the time, and the reality of a non-isothermal model was added. Peebles (1964) continued the calculations and used two equations of state. In one case

* The $30 \%$ error is the sum of two sources of error: the uncertainty in the density data (approx. $15 \%$ ), and the variation in the density due to an optimistic uncertainty in the transition pressure of only 1 Mbar (again 15\% error in density). A 2 Mbar uncertainty in the transition would lead to a total uncertainty in the molecular transition density of $45 \%$. The errors in the metallic density are comparable. 
he used a less dense EOS, by $3 \%$ relative to Stewart's data at $20 \mathrm{kbar}$. That lead to a $25 \%$ lower density at $1.3 \mathrm{Mbar}$ and would require a larger heavy core at the center of Jupiter to provide the necessary total observed mass. It was found that if the hydrogen density were everywhere $2 \%$ greater than the DeMarcus value, no heavy core at all would be needed, otherwise the core constitutes about $3 \%$ of the total mass. This $2 \%$ variation in density is less than the uncertainty in the EOS, even in the metallic phase. Peebles also found that, using the normal DeMarcus EOS extrapolated from Stewart and an adiabatic temperature distribution and a cloud pressure of 1 atmosphere at $150 \mathrm{~K}$, no high density core is required. If the pressure of the observed cloud layer at $150 \mathrm{~K}$ is raised to $5 \mathrm{~atm}$, then $3 \%$ of the mass of the planet is needed as a core. Hence the effect of varying the equation of state of hydrogen by $2 \%$ and the pressure of the cloud layer that is observed both lead to comparable effects as far as their effects on the need for a high density core is concerned.

Lowering the density of the molecular hydrogen allows the molecular to metallic phase transition to occur at a lower pressure which leads to a larger radius and mass of the planet in the higher density metallic phase which in turn diminishes the need for a high density core. The calculated mass of the core in Saturn is considerably larger (approx. 20\%) and varies in the same way.

Now let us consider the size of the metallic hydrogen sphere and its dependence on the EOS. If the model and density distribution of Peebles is used, a variation in the molecular transition density from 0.8 to $1.0 \mathrm{~g} \mathrm{~cm}^{-3}$ (less than the uncertainty), leads to the radius of the metallic sphere $\left(R_{M}\right)$, to vary from 0.84 to 0.79 and the mass fraction from 0.86 to 0.80 , respectively. Hence, a $20 \%$ variation in $D_{t}$ leads to about a $4 \%$ variation in $R_{M}$ and the mass fraction in the metallic phase. In the case of Saturn the same variation in $D_{M}$ allows $R_{M}$ to vary from 0.61 to $0.53(15 \%)$ and the mass fraction from 0.59 to $0.49(10 \%)$. Overall, while these uncertainties are not small, they are probably no more than comparable to the uncertainties arising from the possibly erroneous assumption of a uniform helium distribution throughout the planetary interior.

\section{The Effects of the Unusual Properties of Metallic Hydrogen}

Recently, the speculations on the nature of metallic hydrogen have included its possibly being a high temperature superconductor and/or metastable at pressures below its phase transition. It has been suggested that the possible superconducting nature of metallic hydrogen could lead to the generation of Jupiter's magnetic field (Ashcroft, 1968, Schneider, 1969). Their calculations have concluded that the Debye and critical superconducting transition temperatures could be as high as about $3000 \mathrm{~K}$ and $200 \mathrm{~K}$, respectively. Schneider also found that the critical temperature is lower at both higher and lower pressures compared to the metallic transition pressure, if the interiors of the planets were isothermal and equal to the cloud temperature of $150 \mathrm{~K}$, it would seem feasible. However, at a sufficient depth for the metallic phase to exist, the realistic interior temperature is more likely to be $3000 \mathrm{~K}$ to $4000 \mathrm{~K}$. Hence a superconducting magnetic generator seems unlikely. 
Perhaps the proposed metastability of metallic hydrogen is of interest in terms of the major planets. Because of the possibly high superconducting transition temperature of metallic hydrogen, the question of it possibly being metastable at ambient pressure has been raised. Calculations of metallic hydrogen's crystal structure have been made and indicate the possibility (Schneider, 1969; Brovman et al., 1971). Estimates of its lifetime at zero pressure have been considered as limited by surface evaporation (Chapline, 1972) and by bulk effects (Estrin,' 1971) and found to be probably less than a second, unless a protective environment is provided. In one case it is argued that it cannot be metastable above $14 \mathrm{~K}$ (Liberman, 1972). However it is likely that the lifetime will increase a little (Chapline, 1972) or greatly (Salpeter, 1973) with pressure. At a $100 \mathrm{kbar}$ its lifetime could be considerable. If that is true, it is possible that metallic hydrogen could be present in droplets or as waves on the metallic surface at heights greater than $\boldsymbol{R}_{\boldsymbol{M}}$. If the metallic phase could exist down to a pressure of about $100 \mathrm{kbar}$, then it could be found in the region between 0.8 and 0.96 of Jupiter's radius and between 0.6 and 0.9 in Saturn. In terms of heights, this amounts to about $10^{4} \mathrm{~km}$ for Jupiter and twice that for Saturn. This could be important in considerations about convection and instabilities. The energy content of metastable metallic

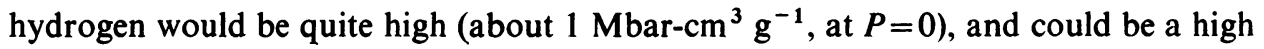
energy density transport mechanism.

Another speculation about metallic hydrogen is that it could be a quantum super fluid (Schneider, 1969), at a pressure of a little more than 100 Mbar. Although Jupiter's central pressure was estimated at one time to be of that magnitude (DeMarcus, 1958) more recent estimates lead to pressures of about 30 to $40 \mathrm{Mbar}$ (Peebles, 1964), hence, it is not likely that such a superfluid is in abundance.

\section{Summary}

In summary, I would like to say that progress is being made in generating EOS data that describe molecular hydrogen and experiments have progressed into the megabar region in the last 2 years and should lead to quite refined data in the next 5 . Probably the importance of this information will be much less than the need for accurate helium-hydrogen phase diagrams, but it appears that area of experimentation is about where megabar pressures were in 1954 when, with great difficulty, pressures of 20 kbar were reached.

The possible spectacular properties of metallic hydrogen should be kept in mind but so far it does not appear any problems of planetary models are better understood by their consideration. Probably the gathering of additional information about the planets via satellites, will be of great stimulus to reduce the uncertainties to a unique model; however, even here on Earth, with the corpus delicti in hand, there is not yet a single agreed upon model.

\section{References}

Abrikosov, A. A.: 1954, Astron. Zh. 31, 112.

Al'tschuler, L. V., Dynin, E. A., and Svidinski, V. A.: 1973, Zh. Experim. Theor. Fiz. 17, 20. 
Ashcroft, N. W.: 1968, Phys. Rev. Letters 21, 1748.

Brovman, E. G., Kagan, Yu., and Kholas, A.: 1971, Zh. Experim. Theor. Fiz. 61, 2429.

Chapline, G. F.: 1972, Phys. Rev. B6, 2067.

DeMarcus, W. C.: 1958, Astron. J. 63, 2.

Dick, R.: 1972, in G. L. Kerley (ed.), High Pressure Physics and Planetary Interiors, North Holland Publ. Co., p. 78.

Estrin, E. I.: 1971, Soviet Phys. JETP Letters 13, 510.

Etters, R. D., Raich, J. C., and Chand, P. J.: 1971, Low Temp. Phys. 5, 6711.

Grigoryev, F. V., Kormer, S. B., Mikhailova, O. L., Tolochko, A. P., and Urlin, V. D.: 1972, JETP Letters 5, 286.

Hawke, R. S., Duerre, D. E., Huebel, J. G., Keeler, R. N., and Klapper, H.: 1972, in G. L. Kerley (ed.), High Pressure Physics and Planetary Interiors, North Holland Publ. Co., p. 44.

Hoover, W. G., Keeler, R. N., Van Thiel, M., Hord, B. L., Hawke, R. S., Olness, R. J., Ross, M., Rogers, F. J., and Bender, C. F.: 1973, Adv. Cryogenic Engin. 18, 447.

Hubbard, W. B.: 1968, Astrophys. J. 152, 745.

Kronig, R., DeBoer, J., and Korringa, J.: 1946, Physica, 12, 245.

Liberman, D. A.: 1972, Los Alamos Sci. Lab., Report LA 4727-MS.

Rogers, F. J., and Neece, G. A., Hoover, W. G.: 1971, J. Compt. Phys. 7, 621.

Østgaard, E.: 1972, Z. Phys. 252, 95.

Peebles, P. J. E.: 1964, Astrophys. J. 140, 328.

Pollack, E. L., Bruce, T. A., Chester, G. V., and Krumhansl, J. A.: 1972, Phys. Rev. 5, 4180.

Ramsey, W. H.: 1950, Monthly Notices Roy. Astron. Soc. 110, 325.

Ross, M.: 1970, Lawrence Livermore Report, UCRL 50911.

Salpeter, E. E.: 1972, Phys. Rev. Letters 28, 560.

Salpeter, E. E.: 1973, Astrophys. J. 181, L83.

Schneider, T.: 1969, Helv. Phys. Acta 42, 957.

Smoluchowski, R.: 1967, Nature 215, 691.

Smoluchowski, R.: 1973, Astrophys J. 185, L95.

Stewart, J. W.: 1956, J. Phys. Chem. Solids 1, 145.

Street, W. P.: 1973, Astrophys. J. 186, 1107.

Trubitsyn, V. P.: 1966, Soviet Phys. Solid State 7, 2708.

Van Thiel, M., Ross, M., Hord, B. L., Mitchel, A. C., Gust, W. A., D’Addario, M. J., Keeler, R. N., and Boutnell, K.: 1972, Phys. Rev. Letters 31, 979.

Wigner, E. P. and Sietz, F.: 1933, Phys. Rev. 43, 804.

Wigner, E. P. and Hunington, H. B.: 1935, J. Chem. Phys. 3, 764.

\section{DISCUSSION}

Hide: Are there any plans to extend the work you have described to hydrogen-helium mixtures?

Graboske: The great difficulty and expense of these high pressure experiments requires that they be carried out on standard materials having a wide range of applications. To my knowledge, there are no current plans to study $\mathbf{H}-\mathrm{He}$ mixtures at such high pressures. However, other materials pertinent to planetary atmospheres, such as methane and ammonia, are being studied at Livermore. Once pure hydrogen and pure helium have been experimentally investigated, I feel it would be important to study $\mathrm{H}$-He mixtures, and high pressure researches in all countries should be encouraged to consider them. I might mention here the recent extensive work by Street at West Point, soon to appear in the Astrophysical Journal, on static studies of the complex phase structure of $\mathrm{H}$-He mixtures at temperatures and pressures appropriate to the giant planet atmospheres. 